\title{
Genome-wide identification and expression analyses of the pectate lyase (PEL) gene family in cotton (Gossypium hirsutum L.)
}

\author{
Huiru Sun ${ }^{1,2}$, Pengbo Hao ${ }^{1,2}$, Qiang Ma², Meng Zhang ${ }^{2}$, Yuan Qin ${ }^{1,2}$, Hengling Wei², Junji Su², Hantao Wang ${ }^{2}$,
} Lijiao $\mathrm{Gu}^{2}$, Nuohan Wang ${ }^{1,2}$, Guoyuan Liü ${ }^{2}$ and Shuxun $\mathrm{Yu}^{1,2^{*}}$

\begin{abstract}
Background: Pectin is a major component and structural polysaccharide of the primary cell walls and middle lamella of higher plants. Pectate lyase (PEL, EC 4.2.2.2), a cell wall modification enzyme, degrades de-esterified pectin for cell wall loosening, remodeling and rearrangement. Nevertheless, there have been few studies on PEL genes and no comprehensive analysis of the PEL gene family in cotton.

Results: We identified 53, 42 and 83 putative PEL genes in Gossypium raimondii (D5), Gossypium arboreum (A2), and Gossypium hirsutum (AD1), respectively. These PEL genes were classified into five subfamilies (I-V). Members from the same subfamilies showed relatively conserved gene structures, motifs and protein domains. An analysis of gene chromosomal locations and gene duplication revealed that segmental duplication likely contributed to the expansion of the GhPELs. The 2000 bp upstream sequences of all the GhPELs contained auxin response elements. A transcriptomic data analysis showed that 62 GhPELs were expressed in various tissues. Notably, most (29/32) GhPELS of subfamily IV were preferentially expressed in the stamen, and five GhPELS of subfamily $\vee$ were prominently expressed at the fiber elongation stage. In addition, qRT-PCR analysis revealed the expression characteristics of 24 GhPELs in four pollen developmental stages and significantly different expression of some GhPELs between longand short-fiber cultivars. Moreover, some members were responsive to IAA treatment. The results indicate that GhPELs play significant and functionally diverse roles in the development of different tissues.
\end{abstract}

Conclusions: In this study, we comprehensively analyzed PELs in G. hirsutum, providing a foundation to better understand the functions of GhPELs in different tissues and pathways, especially in pollen, fiber and the auxin signaling pathway.

Keywords: Gossypium spp., Pectate lyase (PEL), Gene expression patterns, Pollen development, Fiber, Auxin

\section{Background}

Plant growth and development along with cell expansion and division give rise to the morphogenesis of many organs, tissues and cells [1]. The cell wall is a significant structure that maintains the cell's internal pressure, stability, tensile strength and defense [2]. Pectin, which is mainly present in the primary cell wall and middle lamella, is a polysaccharide containing a linear backbone of $\alpha-1,4$-linked galacturonic acid residues that forms a

\footnotetext{
*Correspondence: ysx195311@163.com

${ }^{1}$ College of Agronomy, Northwest A\&F University, Yangling 712100, China

${ }^{2}$ State Key Laboratory of Cotton Biology, Institute of Cotton Research of CAAS, Anyang 455000, China
}

matrix embedded in cellulose and hemicellulose $[3,4]$. Homogalacturonan (HG), a major component of pectin, can be degraded by various pectinases, including the polygalacturonases (PGs), pectin acetylesterases (PAEs), pectin methylesterases (PMEs) and pectate lyases (PELs) [5]. PEL, (EC 4.2.2.2) depolymerizes HG through a $\beta$-elimination reaction, generating 4,5-unsaturated oligosacceharides [6, 7].

PELs have been widely identified in plant pathogenic bacteria, such as Erwinia chrysanthemi, which causes soft-rot disease in many plants [8]. In many higher plants, expression of PEL-like genes has also been found in a variety of tissues, including the 
pollen of tomato, tobacco and Japanese cedar [9-11], ripening fruits of strawberry and banana [6, 12-14], tracheary elements of Zinnia [15], fiber of cotton [16], xylem of poplar [17] and lateral roots of Arabidopsis [18].

In Arabidopsis, a genome-wide analysis and an analysis of promoters showed that PEL-like genes (AtPLLs) play an important role in the development of flowers and cell separation [19, 20]. In pollen development, PELS may regulate the loosening and degradation of the pollen cell wall [11]. In the process of fruit ripening, PELS modify the pectin structure in the cell wall [13]. In Populus, most of the 30 PtPLs are highly expressed in xylem, performing important functions during the development of wood [17]. In Arabidopsis, the increased expression of AtPLAs (PEL genes) promotes the degradation of the pectin-rich middle lamella during lateral root emergence [18]. In rice, the DEL1 gene (a PEL gene, LOC_Os10 g31910) regulates plant growth and leaf senescence through controlling cell numbers and triggering reactive oxygen species accumulation [1]. Auxin, an important plant hormone, regulates plant growth and development by advancing acid-mediated changes in the cell wall [21, 22]. The acidification of the cell wall activates the expansins and PMEs, which causes loosening of the cell wall [2]. Many studies have revealed that PELs respond to IAA treatment $[13,18,19,23,24]$. These studies indicate that PEL genes exhibit extensive functions in plant growth and development and participate in the auxin regulation pathway.

Cotton is the most important natural fiber crop. Pectins are responsible for $25 \%$ of the cell wall components of rapidly elongating cotton fibers (8 days postanthesis (DPA)), indicating that the structure and configuration of pectin can influence fiber quality [25, 26]. A study examining GhPEL indicated that this gene is crucial for the normal elongation of cotton fibers through degradation of de-esterified pectin, facilitating the loosening of the cell wall [16].

However, there have been few studies related to PEL genes in plants, and they have mainly focused on the functional analysis of individual genes. In cotton, most of the $P E L$ genes are unknown. At present, there are no available genome-wide analyses of the cotton $P E L$ gene family. With the completion of the genome sequencing of G. raimondii, G. arboreum and allotetraploid cultivated cotton (G. hirsutum cv TM-l), we can now perform a comprehensive analysis of PELs in cotton [27-31]. In this study, we predicted the PELs of three Gossypium species and analyzed their gene structure, phylogenetic tree, expression characteristics and other features. The results provide a reference for the potential functions of PELs in plant growth and development.

\section{Results}

Genome-wide identification of PELs in G. raimondii, $G$. arboreum and $G$. hirsutum

Based on the conserved Pec_lyase_C (Pfam00544) domain and SMART analyses, we identified 53, 42, and 83 full-length putative PELs in G. raimondii, G. arboreum and G. hirsutum TM-1, respectively. According to their locations on the chromosomes, the family members of the three species were designated GrPEL1 to GrPEL53; GaPEL1 to GaPEL42; and GhPEL1 to GhPEL83, respectively. The lengths of the putative GhPEL proteins varied from 171 (GhPEL20_At) to 680 (GhPEL52_Dt) amino acids (aa), while those of GaPELs ranged from 136 aa (GaPEL36) to 680 aa (GaPEL14), and those GrPELs varied from 222 aa (GrPEL36) to 511 aa (GrPEL27). The predicted Mw, pI, GRAVY and subcellular localization of the protein sequences are shown in Additional file 1: Table S1.

\section{Phylogenetic analysis of the PEL gene family}

To examine the evolutionary relationships of the PEL proteins and classify them into subfamilies according to the established subfamilies in Arabidopsis, we performed a phylogenetic analysis of 285 PEL protein sequences from G. raimondii (53), G. arboreum (42), G. hirsutum (83), Arabidopsis thaliana (26), Corchorus olitorius (16), Theobroma cacao (24), Oryza sativa (11) and Populus trichocarpa (30) to construct an unrooted phylogenetic tree. The PEL proteins were classified into 5 subfamilies (I, II, III, IV and V) (Fig. 1 and Additional file 2: Table S2). Subfamily IV and subfamily V were the two largest subfamilies and contained 101 and 103 PEL members, respectively, while both subfamily I and subfamily III contained only 20 PEL members. The PEL proteins of Oryza sativa, a monocot, were distant from the PEL proteins of the other dicot plants. These results indicated that PELs might have evolved in different directions and expanded to exhibit diverse functions among various species.

\section{Distribution and gene duplication events of PELs}

The chromosomal distributions of GrPELs, GaPELs and GhPELs were determined according to their genomic locations (Additional file 3: Table S3). In G. raimondii, 50 GrPELs were unevenly anchored on 13 chromosomes, while 3 genes (GrPEL51-GrPEL53) were located on scaffolds. D10 contained the most GrPELs (12), followed by D09 with 8 GrPELs. However, there was only one GrPEL on D04 and D12 (Fig. 2a). In G. arboreum, 42 GaPELs were located on 13 chromosomes. Both A06 and A11 contained the most GaPELs (6 each). In contrast, both A05 and A07 only contained one GaPEL (Fig. 2b). A total of 71 GhPELs were mapped to the 25 G. hirsutum chromosomes, with the exception of At02, while 12 


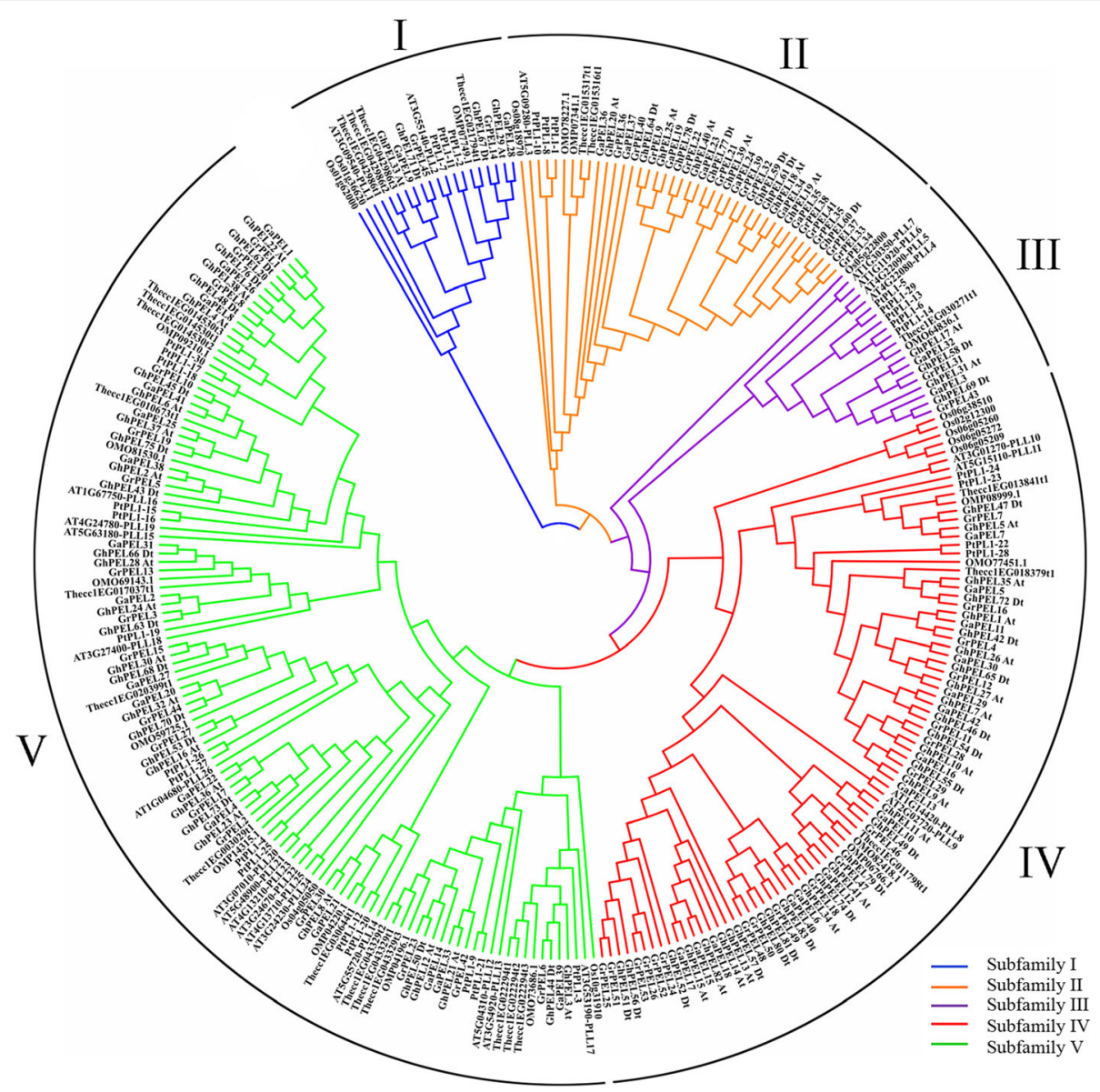

Fig. 1 Phylogenetic tree of PEL proteins. The 285 predicted protein sequences from Gossypium raimondii, G. arboreum, G. hirsutum, Arabidopsis, Corchorus olitorius, Theobroma cacao, Oryza sativa and Populus were aligned with ClustalX 2.0, and the phylogenetic tree was generated using MEGA 6.0 via the neighbor-joining (NJ) method with 1,000 bootstrap replicates. Five subfamilies of PELs are indicated using different line colors

other GhPELs were located on unassembled scaffolds. The distribution of GhPELs on each chromosome was highly uneven: D05 contained the most GhPELs (7); At06 and At09 contained 5 GhPELs; and the other 22 chromosomes contained one to four GhPELs (Fig. 2c).

Previous studies have indicated that gene duplication events are vital to gene family expansion and occur along with plant genome evolution [32]. In the present study, a gene duplication analysis was performed to investigate the expansion mechanism of the PEL gene family in the three Gossypium species. In general, gene duplication events include tandem and segmental duplications. A total of 14, 13 and 35 gene duplication pairs were identified in G. raimondii, G. arboreum and G. hirsutum, respectively, accounting for $71.7 \%, 66,7 \%$ and $86.7 \%$ of the $P E L$ gene family (Additional file 4: Table S4). Based on sequence similarity and chromosomal location, 5, 3 and 5 gene duplication pairs were determined to represent tandem duplication events in G. raimondii, G. arboreum and G. hirsutum, respectively, while 49 other pairs represented segmental duplication events; these pairs are shown in Fig. 3, except for the gene pairs located on unassembled scaffolds. A total of 13 clusters of tandem duplication were located on D08 (GrPEL21/ GrPEL22), D10 (GrPEL32-GrPEL41), D13 (GrPEL48-GrPEL50), D09 (GrPEL24-GrPEL26 and GrPEL28/GrPEL29), A06 (GaPEL17/GaPEL18), A09 (GaPEL23/GaPEL24), A11 (GaPEL34-GaPEL37), At05 (GhPEL13_At-GhPEL15_At), At06 (GhPEL18_At/GhPEL19_At), At09 (GhPEL26_At/GhPEL27_At), Dt05 (GhPEL54_Dt/GhPEL55_Dt) and Dt06 (GhPEL59_Dt-GhPEL61_Dt) (Fig. 2). These results indicated that gene duplication, especially segmental duplication, played an irreplaceable role in the expansion of the PEL gene family in the three Gossypium species.

To investigate the selection pressure for the segmental duplication of PEL gene pairs, the $\mathrm{Ka} / \mathrm{Ks}$ ratio was calculated. The results showed that the $\mathrm{Ka} / \mathrm{Ks}$ ratios for most of the segmental duplications of $P E L$ gene pairs were less than 1.0, indicating that they had experienced purifying selection pressure after gene duplication events (Additional file 5: Table S5). Because of the constraints 


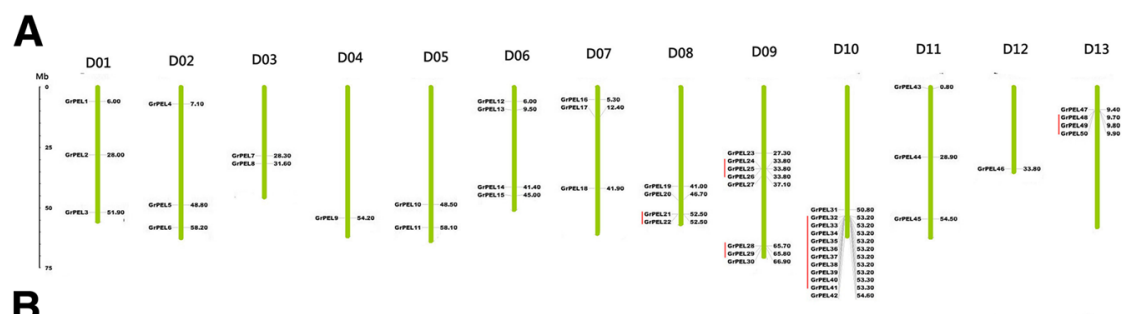

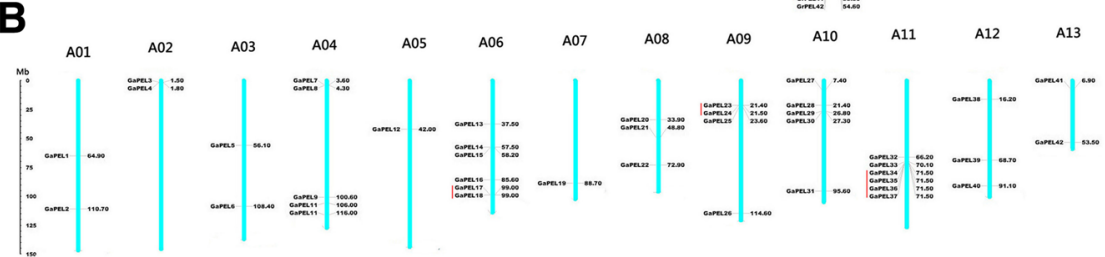
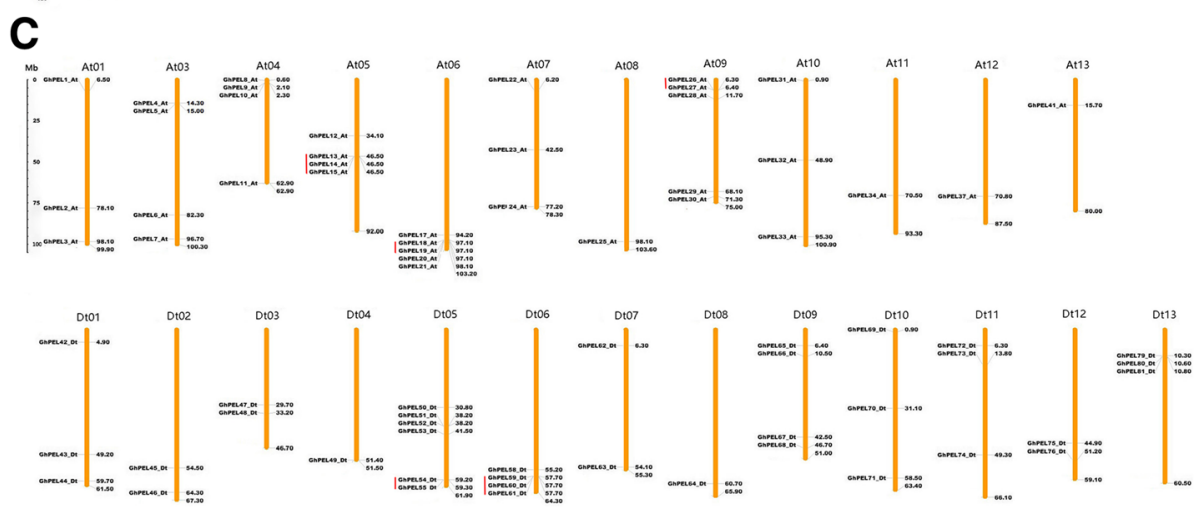

Fig. 2 Chromosomal distribution of PELs from Gossypium raimondii (a), G. arboreum (b), and G. hirsutum (c). The scale represents megabases (Mb). The chromosome numbers are indicated above each vertical bar. The putative PELs are indicated on the different chromosomes. Green, blue and yellow bars represent the physical maps of Gossypium raimondii (a), G. arboreum (b), G. hirsutum (c), respectively. Red lines show the gene pairs involved in tandem duplication

of purifying selection on divergence, most of the segmental duplications of the $P E L$ pairs might exhibit similar functions. Only GhPEL2_At/GhPEL43_Dt presented a $\mathrm{Ka} / \mathrm{Ks}$ ratio greater than 1 , demonstrating that this GhPEL pair had undergone positive selection during cotton evolution.

In addition, divergence time analysis was performed between the segmentally duplicated PEL pairs (Additional file 5: Table S5). In G. raimondii and G. arboreum, the timing of the occurrence of the segmental duplication of $P E L$ pairs was inferred to be $8.48-171.46$ million years ago (MYA), with an average of 91.73 MYA, and 18.35-145.46 MYA, with an average of 85.73 MYA, respectively. In G. hirsutum, the timing of the occurrence of the segmental duplication PEL pairs was presumed to be 1.00-32.75 MYA, with an average of 10.24 MYA.

\section{Conserved domains and amino acid sites of GhPELs}

The Pec_lyase_C domains and signal peptides of the PEL sequences were investigated and shown according to the phylogenetic tree of the GhPELs (Additional file 6:
Figure S1). All of the GhPELs contained a Pec_lyase_C domain, indicating that this domain was conserved. Four Asp residues, one Cys residue, one Arg residue, and five additional amino acid residues (Asp, His, Thr, Pro and Arg) in the Pec_lyase_C domain involved in $\mathrm{Ca}^{2+}$-binding, disulfide bonds, catalysis and substrate binding, respectively, were found to be highly conserved, indicating that these amino acid sites were significant for the function of GhPELs (Additional file 7: Figure S2). Most members of the GhPELs (77.1\%) exhibited the predicted signal peptide. However, the members of subfamily I exhibited no signal peptide, which was consistent with a previous study on PtPLs [17].

\section{Gene structure and conserved protein motifs of GhPELs}

To further understand the conservation and diversification of the GhPELs, their exon-intron structures and conserved motifs were investigated and were shown in Fig. 4. The members of subfamily I and subfamily III each contained 5 exons and 3 exons, respectively. Most members of subfamily II exhibited 2 exons, except for GhPEL20_At, GhPEL19_At and GhPEL60_Dt, which 


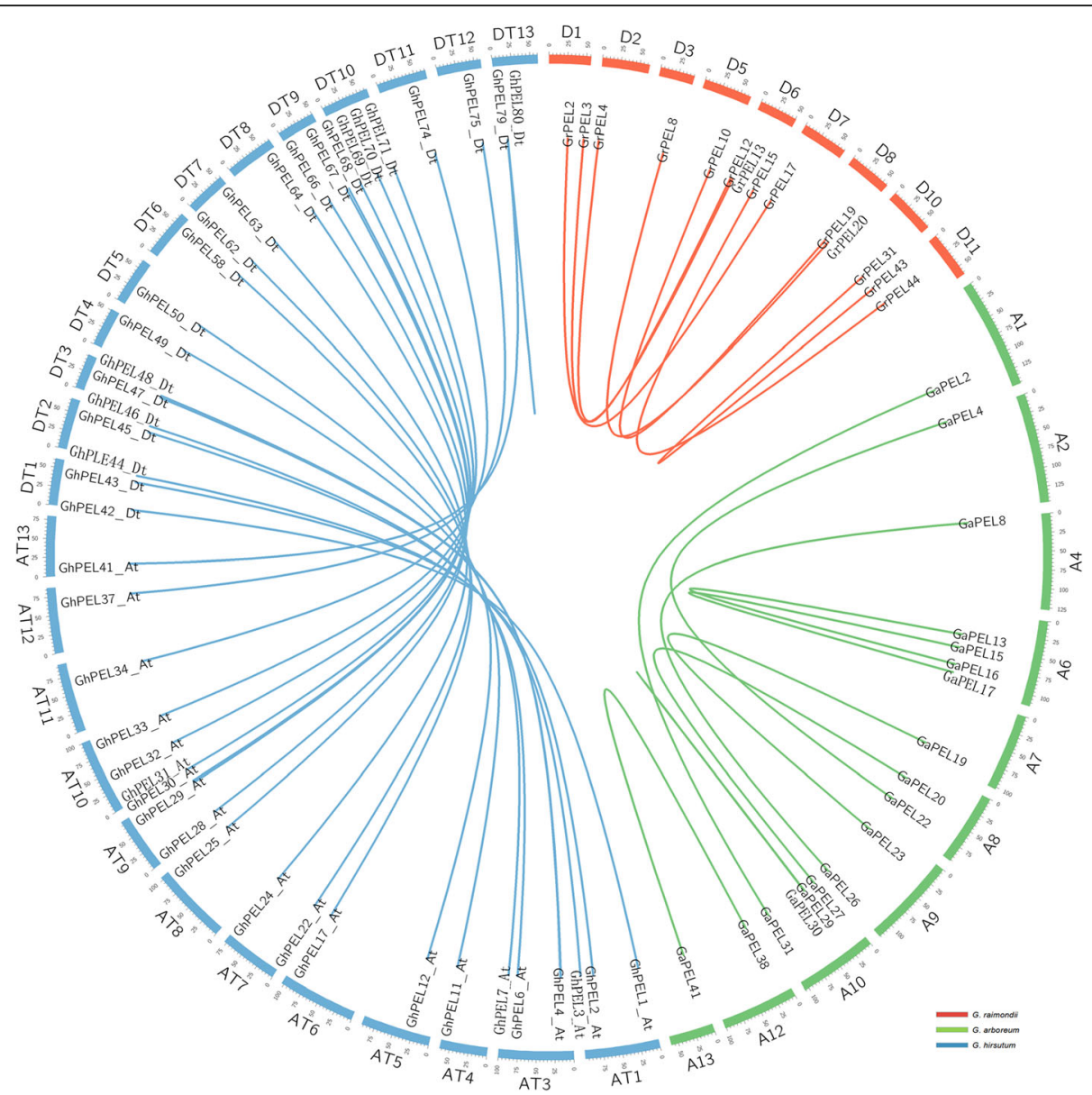

Fig. 3 Circos figure of gene pairs of segment duplication in GrPELs, GaPELs and GhPELs. The chromosomes of Gossypium raimondii, G. arboreum and G. hirsutum are filled with red, green, and blue, respectively. Gene pairs involved in segment duplication are linked by a line

presented 3 exons. More than half (17/32) of subfamily IV contained 4 exons, while the other members displayed 2 or 3 exons, with the exception of GhPEL52_Dt, with 7 exons. The exon numbers of subfamily $\mathrm{V}$ members varied from 3 to 7 . The exon numbers were highly diverse among GhPELs (ranging from two to seven), indicating that functions of GhPELs might be diverse. However, closely related GhPELs showed similar exon-intron structures, and these genes might play similar roles in plant growth and development (Fig. 4b).

We identified 6 conserved motifs of the GhPEL proteins using MEME (Fig. 4c). Within a given subfamily, most of the members exhibited similar motif construction. Motifs 1, 5, 3 and 6 within the conserved Pec_lyase_C domain were identified in most of the GhPEL proteins. A total of $90.4 \%$ of the GhPEL proteins (except for the members of subfamily I and GhPEL20_At, GhPEL40_At, GhPEL19_At, and GhPEL43_Dt) contained motif 4. Motif 2 existed in $96.4 \%$ of the GhPEL proteins (except for GhPEL20_At, GhPEL40_At, and GhPEL43_Dt). Taken together, the motifs and their arrangement showed a high conservation in the GhPEL family.

\section{Analysis of cis-elements related to auxin in putative GhPEL promoter regions}

Many studies have shown that PELs respond to auxin treatment. Therefore, the $2000 \mathrm{bp}$ upstream regions from the initiation codons (ATG) of GhPELs were scanned in the PLACE database to obtain the cis-acting elements related to auxin. The results showed that all of the putative GhPEL promoter regions contained at least one of the six major auxin-responsive cis-elements: S000024, S000026, S000270, S000273, S000360, and S000370. In addition, the putative promoter region of GhPEL63_Dt contained the largest number (15) with all six auxin-responsive cis-elements. $\mathrm{Ca}^{2+}$ - mediated crosslinking of demethyl-esterified HG, the substrate of PEL, can change cell wall structure, which is important for cell expansion and division [33]. A $\mathrm{Ca}^{2+}$-responsive cis-element (S000501) and calmodulin-binding/CGCG box (S000507) were identified in $35(42.2 \%)$ of the 

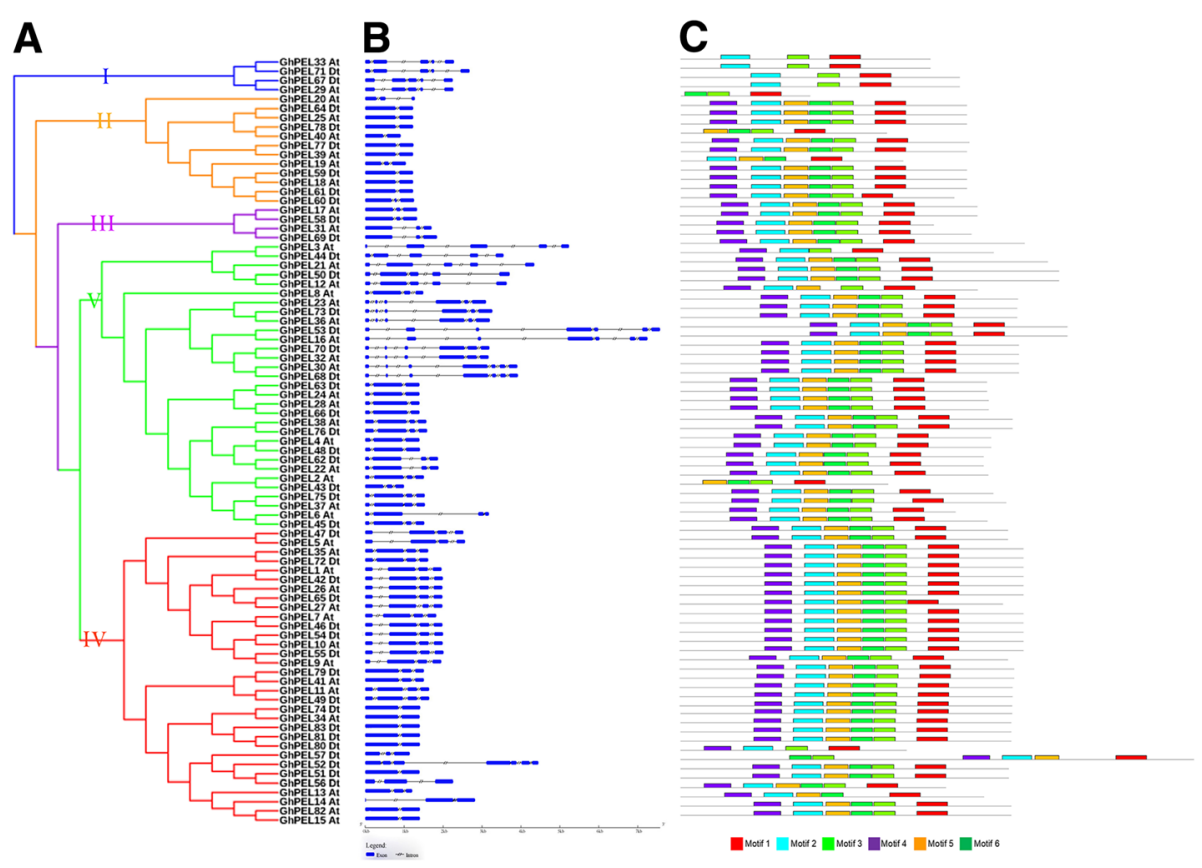

Fig. 4 Phylogenetic relationships, gene structure and motif analysis of GhPELs. a Phylogenetic analysis of GhPEL proteins using MEGA 6.0 via the neighbor-joining $(\mathrm{NJ})$ method with 1,000 bootstrap replicates. b Exon-intron organization of the GhPEL family. The exons and introns are indicated with blue filled boxes and black lines, respectively. c Six motifs of GhPEL proteins determined using MEME. Different colors represent different motifs

putative promoter regions of GhPELs (Additional file 8: Table S6). This result indicated that some GhPELs might alter the configuration of the cell wall with $\mathrm{Ca}^{2+}$.

\section{Expression patterns of GhPELs in different tissues}

To explore the possible biological functions, the spatio-temporal expression patterns of GhPELs were investigated in different tissues, including root, stem, leaf, petal, stamen, pistil, ovules and fibers at various developmental stages. Using the transcriptome datasets of G. hirsutum (TM-1) [30], the expression profiles of 62 GhPELs with FPKM $\geq 1$ in at least one of the 8 investigated tissues were shown in Fig. 5. The other 21 GhPELs, including all of the members (12) of the subfamily II, were very low or not expressed in all of the investigated tissues and developmental stages and 15 genes from gene duplication events, indicating that functional redundancy or pseudogenes existed in the GhPEL family.

Based on a clustering analysis, the 62 GhPELs were divided into 4 major patterns (A-D) (Fig. 5). Cluster A contained 29 genes, all belonging to subfamily IV and dominantly expressed in the flower, especially in the stamen, with very low expression in other tissues. In cluster B, all 4 GhPELs (GhPEL28_At, GhPEL37_At, GhPEL63_Dt and GhPEL66_Dt) from subfamily $\mathrm{V}$ were shown to have higher expression levels in the leaf than in other tissues. Cluster C contained 16 GhPELs from subfamily V and I general expression in all of the tissues, with some genes primarily expressed in fiber, stamen and other tissues. Cluster D was composed of another 13 GhPELs, from subfamily V and III, with lower expression in fiber than vegetative and reproductive organs. The gene expression pattern could manifest functions of genes, in part. The dominant expression of GhPELs in cluster A indicated that these genes performed crucial and conserved functions in the development of the flower, especially the stamen, which was concordant with PLLs generally expressed in flower and several PLLs highly expressed in pollen in Arabidopsis [19]. Some GhPELs in cluster C, including the GhPEL gene (GhPEL48_Dt) that was reported to regulate fiber elongation [16], showed higher expression in fiber than other tissues, which hinted that these GhPELs might regulate fiber development.

\section{Expression characterization of GhPELs in anther development}

To explore the expression features of GhPELs in anther development, using qRT-PCR, we identified the expression of 24 GhPELs in four stages of anther development, including meiosis stage, mononucleate stage, binucleate stage and mature stage (Fig. 6). The expression levels of 7 GhPELs (GhPEL29_At, GhPEL31_At, GhPEL62_Dt, GhPEL6_At, GhPEL21_At, GhPEL17_At and GhPEL58_Dt) were highest in the meiosis stage and decreased in subsequent stages, especially GhPEL17_At and GhPEL58_Dt with plummeting 


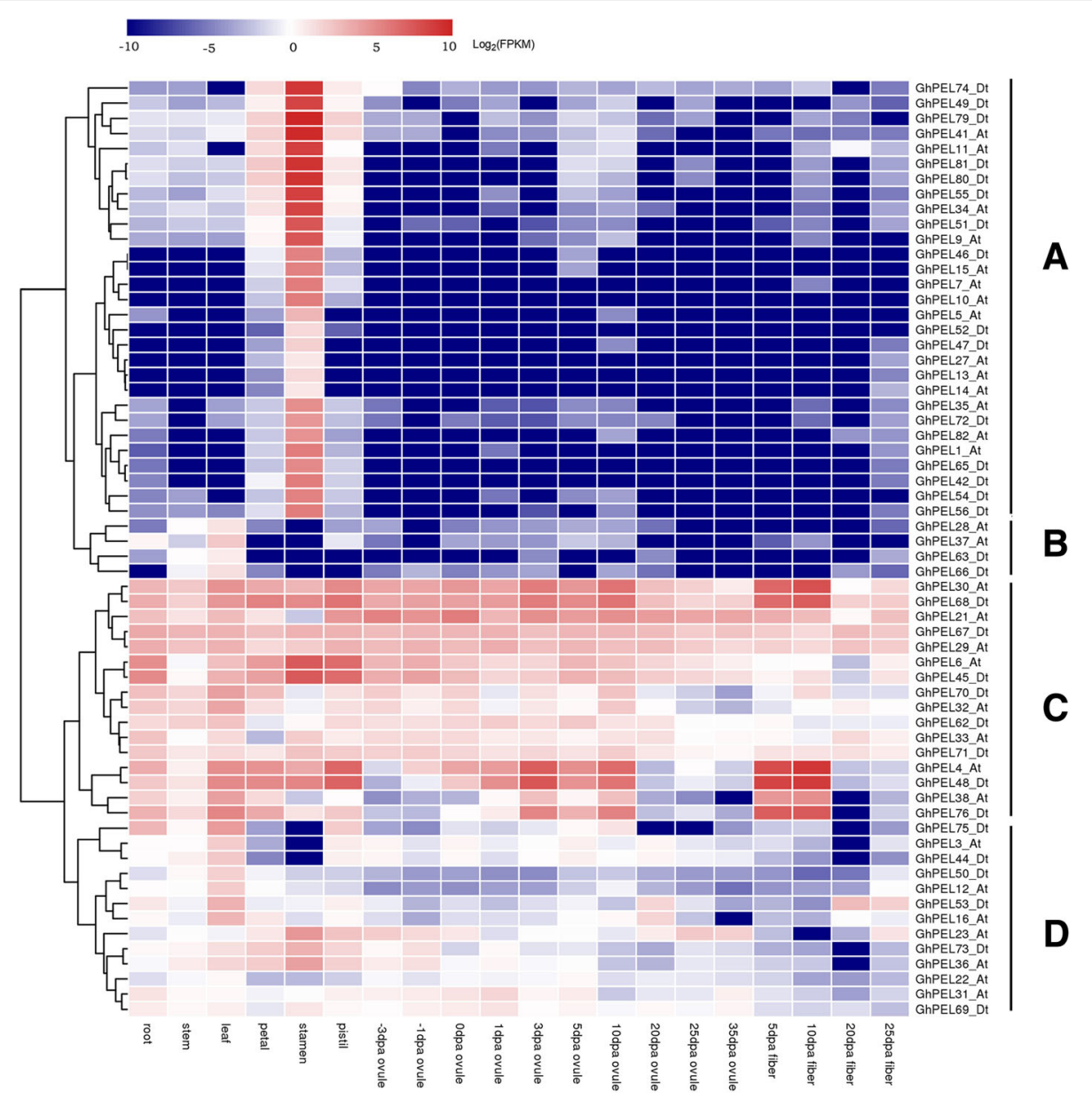

Fig. 5 Expression profiles of GhPELs in different tissues. The tissues are shown on the bottom; the genes are shown on the right; and the phylogenetic relationships are shown on the left and have been classified into four groups (a-d). Scale bars at the top represent log $2^{-}$ transformed FPKM values. -3 DPA to 35 DPA indicates $-3,-1,0,1,3,5,10,20,25$ and 35 days after anthesis

expression after the meiosis stage (Fig. 6a). Twelve GhPELs showed a constantly increasing expression to a peak at the binucleate stage, then decreased at the mature stage (Fig. 6b). The remaining 5 genes (GhPEL5_At, GhPEL7_At, GhPEL22_At, GhPEL36_At and GhPEL45_Dt) exhibited higher levels at the mature stage than at the other three stages, especially GhPEL36_At, which displayed a very low expression at the other three stages except for the mature stage (Fig. 6c). These results indicated that some GhPELs performed various and significant functions in the different development stages of anthers.

\section{Characterization of GhPEL gene expression in fiber development}

Two cultivars with significantly different fiber lengths (TM-1, with longer fibers, and kenN27-3, with shorter fibers) were chosen to explore the function of GhPELs in fiber development [34]. The expression of 7 genes (GhPEL4_At, GhPEL48_Dt, GhPEL30_At, GhPEL68_Dt,
GhPEL76_Dt, GhPEL38_At, and GhPEL53_Dt), most of which exhibit higher expression in fiber than other organs, was analyzed in 10 DPA, 15 DPA, 20 DPA and 30 DPA fibers of TM-1 and kenN27-3. The results revealed that 5 genes (GhPEL4_At, GhPEL48_Dt, GhPEL30_At, GhPEL68_Dt and GhPEL76_Dt) displayed higher expression levels in TM-1 than in kenN27-3 in all stages and showed a significant difference in at least one stage, especially in the rapid elongation stage of fiber (10 DPA and 15 DPA) (Fig. 7a). GhPEL38_At and GhPEL53_Dt exhibited remarkably higher expression in fiber elongation stages and lower expression in the secondary wall thickening stage in TM-1 than kenN 27-3 (Fig. 7b). According to these results, these GhPELs might play an important role in fiber elongation.

\section{Responses of GhPEL genes to IAA treatment}

Studies have shown that auxin can regulate the activity of PEL $[18,19,23]$. Therefore, the expression features of 


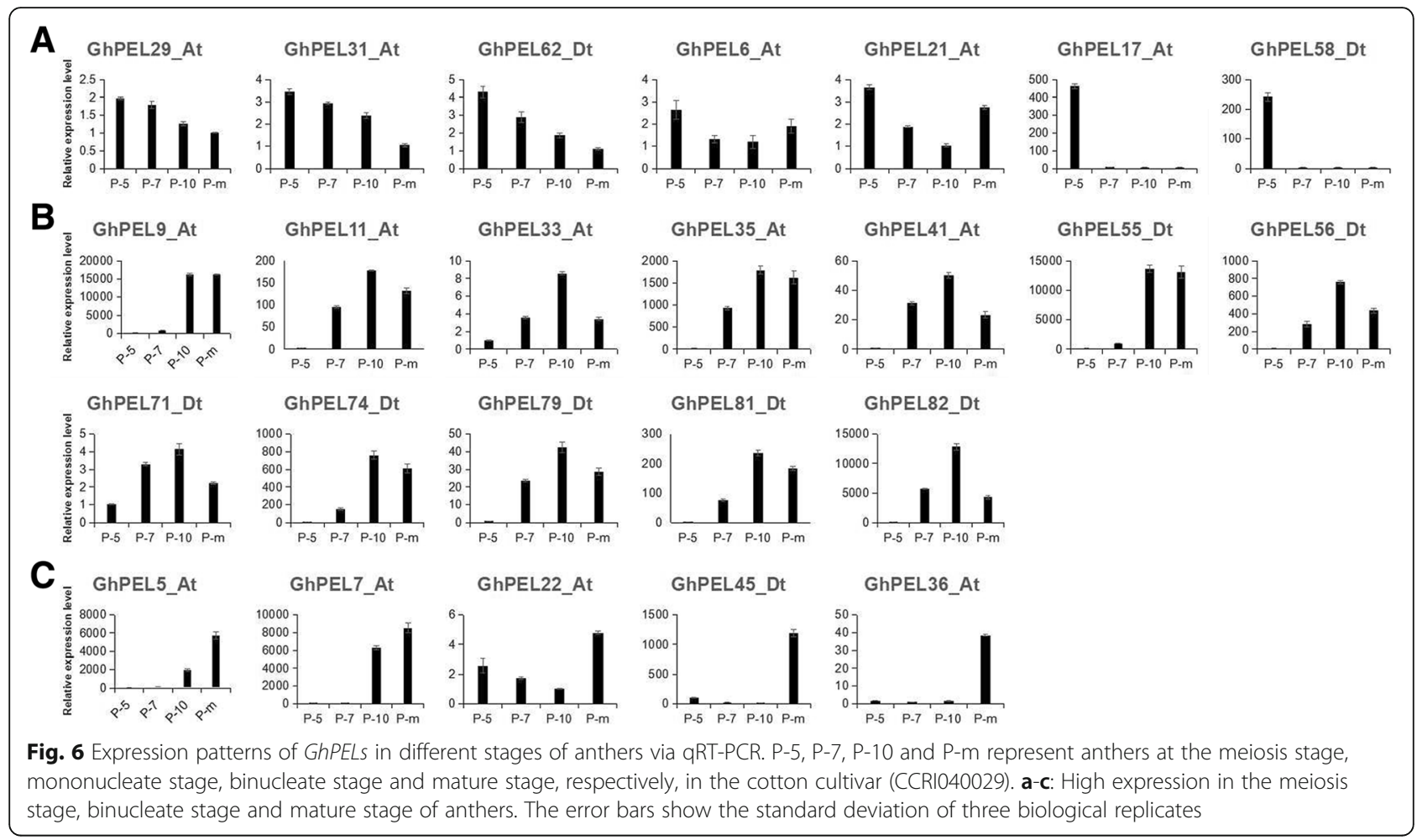

16 GhPELs were explored after IAA treatment (Fig. 8). Seven genes were notably up-regulated, and eight genes were notably down-regulated, mainly at $6 \mathrm{~h}$ and $12 \mathrm{~h}$ (Fig. 8a, b). The expression of GhPEL67_Dt showed notably earlier down-regulation and then up-regulation in response to IAA treatment at $3 \mathrm{~h}$ and $6 \mathrm{~h}$, respectively (Fig. 8c). These results indicated that some GhPELs might take part in the biological pathways regulated by auxin.

\section{Discussion}

\section{Characterization of PEL genes in cotton}

Previous studies on PEL genes have mainly been conducted in plant pathogenic bacteria, focusing on pathogenic mechanisms $[8,35]$. However, PEL-like genes have also been found in many plants, with genome-wide analyses revealing 26 PEL homologous genes in Arabidopsis, 12 in Oryza sativa, 46 in Brassica rapa and 30 in Populus trichocarpa $[17,19,20,36]$. In the present study, a

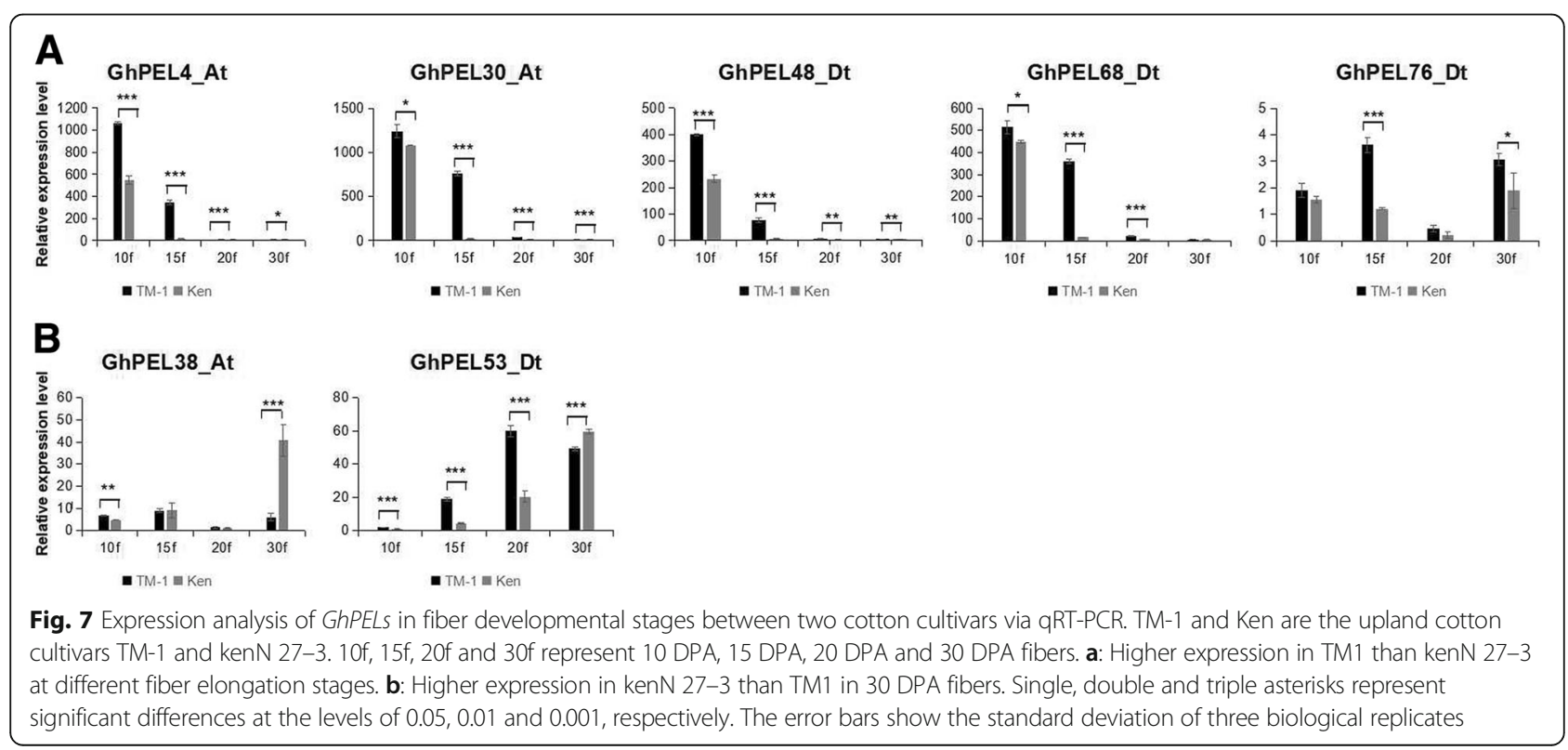



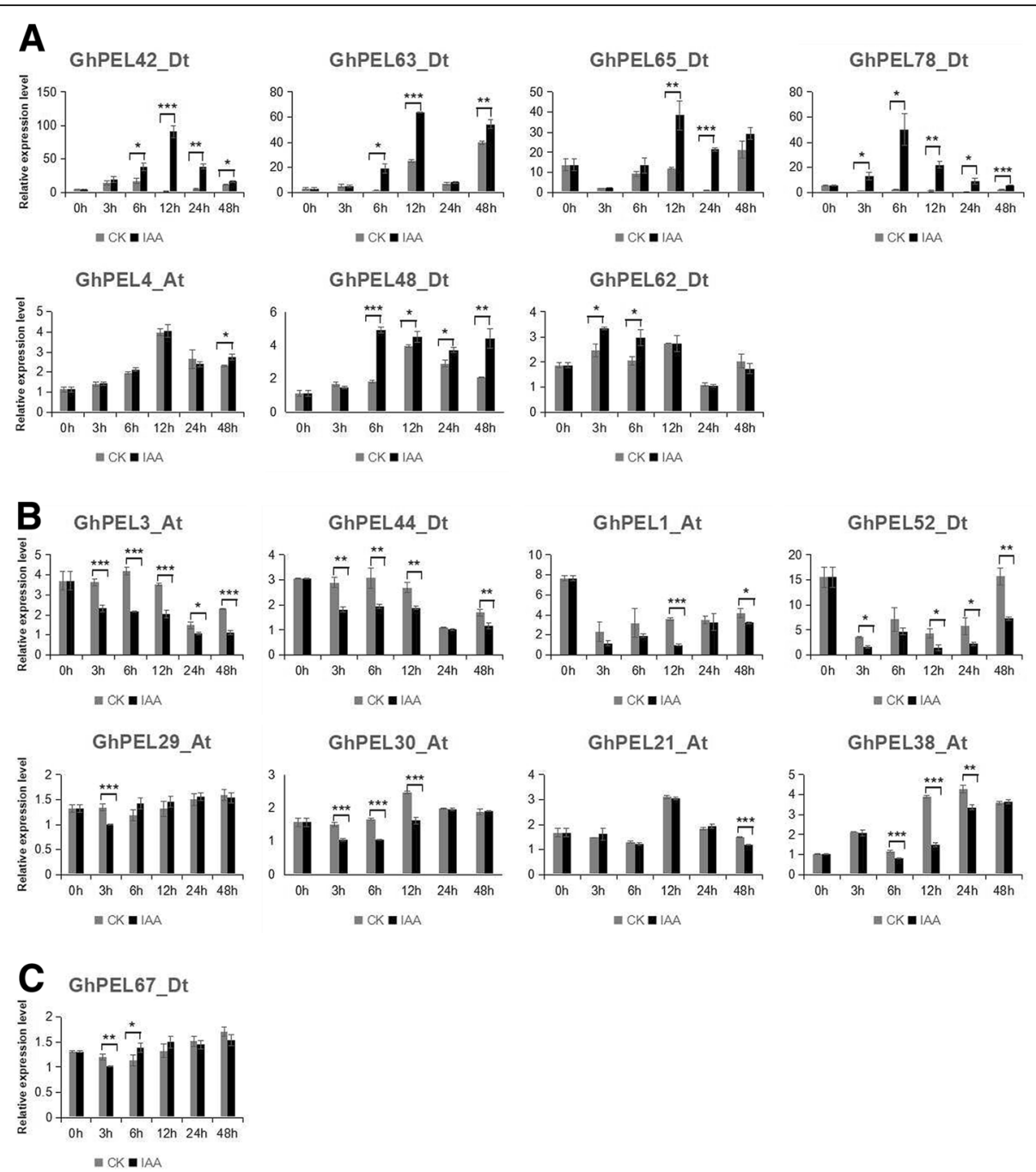

Fig. 8 Expression analysis of GhPELs in the leaves under IAA treatment via qRT-PCR. 0 h, 3 h, 6 h, 12 h, 24 h and $48 \mathrm{~h}$ indicate hours after IAA treatment. a: Higher expression under IAA treatment than in CK at different IAA treatment times. $\mathbf{b}$ : Lower expression under IAA treatment than in CK at different IAA treatment times. c: No difference in expression between IAA treatment and CK at all IAA treatment times. Single, double and triple asterisks represent significant differences at the levels of 0.05, 0.01 and 0.001, respectively. The error bars show the standard deviation of three biological replicates

smaller number of PEL genes was identified in Oryza sativa (12 to 11), ignoring the Os06 g38520 genes due to exceeding the threshold (1e-10). We also identified 53 $P E L$ homolog genes in G. raimondii, 42 in G. arboreum and 83 in G. hirsutum; these numbers are larger than those reported in most other species, indicating that gene expansion has occurred in the PEL gene family, especially in the IV and V subfamilies, during the evolution of cotton genomes. Gene duplication events have been reported to play an important role in gene expansion and both functional conservation and novelty in gene families [37, 38]. Our analysis of gene duplication in three cotton species showed that gene expansion in $G$. hirsutum and G. arboreum likely resulted primarily from segmental duplication (72.3\% and $47.6 \%)$, whereas in G. raimondii, it resulted almost equally from tandem and segmental duplication (Additional file 5: Table S5). Genomic studies of cotton have revealed that two whole-genome duplication (WGD) events occurred in both G. arboreum and G. raimondii, one ancient and one recent event, at approximately $115-146$ and 13-20 MYA, respectively [27-29]. Subsequently, polyploidization of A and D diploids and the emergence of G. hirsutum occurred, approximately 1-2 MYA [30]. In our study, analysis of the timing of the occurrence of segmental duplications showed that most of the events observed in G. raimondii and G. arboreum might have occurred from 8.48-145.46 MYA, except for GrPEL3/ GrPEL13 (148.15 MYA) and GrPEL31/GrPEL43 (171.46 MYA), suggesting that these duplication events might 
have derived from the two WGD events. In G. hirsutum, the segmental duplication events most likely occurred before the hybridization of the two extant ancestors, according to the range of divergence times (1.00-32.75 MYA) and average time (10.24 MYA). The uneven chromosomal distribution of the $P E L$ genes in the three cotton species might be due to the occurrence of these gene duplication events in the evolution of cotton.

Phylogenetic analysis indicated that the PEL genes could be classified into five subfamilies, with the largest members in subfamilies IV and V (Fig. 1, Additional file 2: Table S2). The PELs of Oryza sativa were located a long distance from those of the other plants, which might be related to different functions of these proteins between monocots and dicots [39]. All of the GhPELs exhibited the conserved Pec_lyase_C domain, with highly conserve $\mathrm{Ca}^{2}$ ${ }^{+}$-binding, disulfide bonds, catalysis and substrate binding amino acid sites (Additional file 6: Figure S1 and Additional file 7: Fig. S2). The conserved motif analysis showed that the six motifs existed in most of the GhPELs (Fig. 2c). These data indicated that the GhPELs (especially the members of the same subfamily) might show a relatively conserved function in upland cotton growth. However, gene structure analysis revealed high diversity in the exon numbers of GhPELs, which could be related to diversification of their functions (Fig. 2b) [19, 40]. The similarities and differences of the gene structures, domains and motifs of GhPELs might be related to conservation and subfunctionalization, resulting from their long evolutionary history and gene duplication in cotton $[37,41]$.

\section{Role of GhPELs in plant growth and development}

PELs are cell wall-modifying enzymes that can cleave $\alpha-1,4$-glycosidic linkages of demethylated HG, the structural polysaccharide of the primary cell walls and middle lamella in higher plants, via a $\beta$-elimination mechanism [5]. Highly methyl-esterified HG is secreted into the extracellular matrix during cell division and is then de-esterified by PMEs. Demethylated HG is degraded by PELs and PGs more easily, promoting the loosening of the cell wall and regulating cell growth, cell division and organ morphogenesis in plant growth and development $[2,42,43]$. These findings indicate that pectinases, including PGs, PAEs, PMEs and PELs, regulate the cell wall composition and structure, playing important roles in different developmental processes of plants.

Comprehensive analyses of PEL genes have been reported in Arabidopsis, Brassica rapa and Populus, with most of these genes being expressed in flowers $[9,10$, $17,19,20,36]$. In the present study, a transcriptomic analysis of GhPELs revealed that $90 \%$ of the members in subfamily IV were preferentially expressed in the stamen, in agreement with the above reports (Fig. 5). To further explore the differences in the expression of
GhPELs in anther development, we analyzed the expression of 24 GhPELs in four developmental stages of anthers, with 7, 12 and 5 GhPELs showing peak expression levels in meiosis, binucleate and mature stages, respectively (Fig. 6). These results indicated that many GhPELs performed synergistic and diverse functions in the process of anther development in upland cotton. Similar findings have been reported in Brassica rapa, in which BrPLL1, BrPLL8-1 and BrPLL11-3 were expressed only at the mature pollen stage, while BrPLL6 showed specific expression at the meiosis and tetrad stages of anthers, and in tomato, in which LAT56 and LAT59 displayed maximal expression levels in the mature anthers $[9,36]$. The PELs expressed during anther development might function to facilitate the degradation of the primary cell wall in pollen mother cells (PMC) during the meiosis stage and take part in anther dehiscence, cell wall loosening in pollen, pollen tube elongation and the promotion of pollen penetration through style tissue degradation in mature anthers [11, 44-46].

Cotton fiber, which is a widely used natural fiber in the textile industry, is produced from a single differentiated epidermal cell of the ovule [47, 48]. In cotton fiber, pectin was produced beginning at anthesis, continuously through 19-20 DPA, and was only found only in the primary cell wall [49]. Here, we explored the expression features of 7 GhPELs, including 3 paralogous gene pairs with high expression in fiber, in two upland cotton cultivars showing significantly different fiber lengths. All 7 GhPELs exhibited significantly higher levels of expression in TM1 than kenN 27-3 during the rapid elongation stage of fiber (Fig. 7). However, GhPEL38_At and GhPEL53_Dt showed lower expression at $30 \mathrm{DPA}$, which was the secondary wall thickening stage. GhPEL (GhPEL48_Dt) has been shown to play an important role in fiber cell elongation through the degradation of pectin in the primary cell wall, facilitating cell wall loosening [16]. GhPEL4_At, GhPEL30_At, GhPEL68_Dt and GhPEL76_Dt appear to exhibit the same expression characteristics as GhPEL48_Dt, indicating that these genes might perform similar functions in fiber elongation. GhPEL38_At and GhPEL53_Dt showed expression levels at the secondary wall thickening stage that were different from those in the other 5 GhPELs, which is probably related to their different roles in secondary wall thickening in fiber. A study of PtPLs revealed that a PtPL1-18 overexpression line exhibited much thinner secondary walls than control plants, which indicated that PELs could regulate the structure of secondary walls as well as primary cell walls [17]. All of these results indicated that these genes played a substantial role in fiber elongation and influence the quality of fiber in upland cotton. 
Auxin is involved in acid-mediated changes in the cell wall, inducing the expansins and pectinases, leading to cell wall loosening and organ initiation [21, 50]. Previous reports have revealed that $P E L$ genes take part in pathway responses to auxin to regulate cell elongation and differentiation and other plant developmental processes $[18,19,24]$. According to our analysis of cis-elements, all of the GhPELs contained at least one of the six auxin-responsive cis-elements (Additional file 8: Table S6). Therefore, we randomly chose 16 GhPELs to investigate the response to IAA treatment. All of these genes showed a response to IAA treatment, primarily at $6 \mathrm{~h}$ and $12 \mathrm{~h}$ (Fig. 8). Among these genes, GhPEL30_At, GhPEL38_At and GhPEL48_Dt also participate in the development of fiber, and GhPEL62_Dt is involved in anther development, especially at the meiosis stage. Previous studies have shown that auxin regulates the development of fiber elongation and the anther at early and late stages [51-53]. Our results revealed that GhPELs likely regulate plant growth and development by responding to the IAA signal.

Previous studies have shown that $P E L$ genes play significant roles in different plant development processes, mainly by changing the cell wall composition and structure $[1,5]$. PEL and pectin methylesterases can alter the cell wall composition, cell wall structure and cell wall loosening by regulating the content and status of methyl-esterified and de-esterified HG [54], thereby affecting plant morphology [1] fruit firmness, pathogen resistance [14], anther development [36] and fiber elongation [16]. Moreover, auxin participates in molding the cell wall and could regulate PEL enzyme activity [24]. According to our gene expression analysis, GhPELs showed diverse gene expression features in different tissues. In particular, we analyzed the expression of GhPELS in anther and fiber development and in response to IAA treatment and inferred that they played roles in these processes likely by altering cell wall composition, cell wall structure and cell wall loosening. However, the regulatory networks and functions of the GhPELs require further studies.

\section{Conclusions}

We performed a genome-wide analysis of the PEL gene family in G. raimondii, G. arboreum, and G. hirsutum. According to analyses of phylogeny, chromosomal location and gene duplication events, PEL genes were divided into 5 subfamilies, and it could be inferred that $P E L$ gene expansion occurred due to gene duplication. All of the GhPELs exhibited the conserved Pec_lyase_C domain, and diverse gene structures were observed among them. The analysis of expression revealed that the GhPELs showed different expression features in different organs and developmental stages and could respond to IAA treatment. These results indicated that PELs played important roles in plant growth and development, especially in anther and fiber development and the auxin signaling pathway. The results of our study provide a fundamental basis for further research on the functions of $P E L$ genes in cotton.

\section{Methods \\ Identification of PELs in cotton}

The conserved Pec_lyase_C (Pfam00544) domain was downloaded from Pfam (http://pfam.xfam. org) [55] and used to search against predicted proteins of G. raimondii (JGI_v2.1), G. arboreum (BGI_v2.0) and G. hirsutum acc. TM-1 (NAUNBI_v1.1) from the CottonGen website (https://www.cottongen.org/icgi/home), using HMMER 3.0 [56]. We also searched the Arabidopsis genome (TAIR 10, http://www.arabidopsis.org) using the Pec_lyase_C domain. The E-value threshold for the HMMER search was set at $1 \mathrm{e}-10$ to obtain possible PEL proteins. Then, the normal mode of the SMART database (http://smart.embl-heidelberg.de/) was used to confirm every putative GhPEL protein with a Pec_lyase_C domain [57]. Only the sequences containing a conserved Pec_lyase_C domain were employed for further analysis.

The theoretical molecular weight $(\mathrm{Mw})$, isoelectric point (pI), grand average of hydropathicity (GRAVY) and subcellular localization of the predicted GhPELs were predicted using ExPASy (http://cn.expasy.org/tools) and the CELLO v2.5 server (http://cello.life. nctu.edu.tw/) $[58,59]$.

\section{Sequence alignment and phylogenetic analysis}

Multiple alignment of all the predicted PEL protein sequences from the three Gossypium species, Arabidopsis, Corchorus olitorius, Theobroma cacao, Oryza sativa and Populus genomes was performed using ClustalX 2.0 [60]. An unrooted phylogenetic tree was generated using the neighbor-joining $(\mathrm{NJ})$ method and the amino acid p-distance model in MEGA 6.0 [61]. Bootstrap resamplings (1000) were used to assess the reliability of interior branches.

\section{PEL gene locations on cotton chromosomes and gene duplication analysis}

The physical chromosome locations of all PEL genes were obtained from the genome sequence databases of the three Gossypium species and visualized with MapInspect (http://www.plantbreeding.wur.nl/uk/ softwaremapinspect.html). The predicted PEL proteins of the three cotton species were aligned with ClustalW2 at EMBL-EBI (http://www.ebi.ac.uk/Tools/msa/clustalw2/). Gene duplication was confirmed if the following conditions were satisfied: (1) the coverage of the alignment was $>80 \%$ of the longer gene; (2) the identity of the 
aligned regions was $>80 \%$; and (3) genes separated by five or fewer gene loci with a distance of less than $100 \mathrm{~kb}$ on the same chromosome were considered to represent a tandem duplication [62-64]. A diagram of segmental duplication was drawn with Circos 0.69 [65]. Nonsynonymous (Ka) and synonymous substitution (Ks) rates were calculated using DnaSp V5.0 software, employing the full-length gene sequences of the segmental duplicated PEL gene pairs from the three cotton species aligned by ClustalX 2.0 [66]. The $\mathrm{Ka} / \mathrm{Ks}$ ratio was assessed to determine the molecular evolutionary rates of each gene pair. In general, $\mathrm{Ka} / \mathrm{Ks}<1$ indicates purifying selection; $\mathrm{Ka} / \mathrm{Ks}=1$ indicates neutral selection; and $\mathrm{Ka} / \mathrm{Ks}>1$ indicates positive selection. The divergence times of these gene pairs were estimated using the formula " $\mathrm{t}=\mathrm{Ks} / 2 \mathrm{r}$ ", with $\mathrm{r}\left(2.6 \times 10^{-9}\right)$ representing neutral substitution $[30,67]$.

\section{Multiple sequence alignments and conserved Pec_lyase_C domain analysis}

The GhPEL protein sequences were employed for multiple sequence alignments with ClustalX 2.0. SMART was applied to determine the conserved Pec_lyase_C domains. The SignalP 4.0 server (http://www.cbs.dtu.dk/ services/SignalP/) was used to predict potential signal peptides within the GhPEL proteins (cutoff $>0.45$ ) [68].

\section{Gene structure and conserved motif analysis}

The genomic sequences and positions of the exons and introns of GhPELs were employed to visualize PEL exon-intron structures on the Gene Structure Display Server (GSDS) (http://gsds.cbi.pku.edu.cn/) [69]. The MEME program was used to analyze the conserved motifs of the GhPEL protein sequences with the following parameters: site distribution, zero or one occurrence per sequence; number of motifs, 6; and motif width, 6-50 [70].

\section{Analysis of cis-elements of upstream sequences}

To determine the cis-elements of the predicted promoters, the $2000 \mathrm{bp}$ genomic DNA sequences upstream of the initiation codon (ATG) of all GhPELs were employed to search the PLACE database (http:// www.dna.affrc.go.jp/PLACE/signalscan.html).

\section{Gene expression pattern analysis}

The expression levels of GhPELs in different tissues were obtained from previously reported transcriptome data [30]. The GhPELs with an FPKM >1 were used for further expression analysis [71-73]. The expression clusters were calculated using Mev4.6.2 software (http:// www.tm4.org/mev.html).
Plant materials and treatments

Three G. hirsutum cultivars (TM-1, kenN 27-3 and CCRI040029) were field grown in Anyang, Henan province, China. The fibers were separated from the ovules at 10, 15, 20 and 30 DPA. The anthers of CCRI040029 were harvested when the flower buds had grown to $5 \mathrm{~mm}, 7 \mathrm{~mm}$ and $10 \mathrm{~mm}$ and at anthesis [74].

TM-1 was also grown in a climate-controlled greenhouse (light/dark cycle: $16 \mathrm{~h}$ at $28{ }^{\circ} \mathrm{C} / 8 \mathrm{~h}$ at $22{ }^{\circ} \mathrm{C}$ ) and was employed to investigate the responses to IAA treatment. Seedlings exhibiting third true leaves were sprayed with $100 \mathrm{mM}$ IAA and water as a control group. The leaves of ten seedlings in each group were collected at $0 \mathrm{~h}, 3 \mathrm{~h}, 6 \mathrm{~h}, 12 \mathrm{~h}, 24 \mathrm{~h}$ and $48 \mathrm{~h}$ after treatment. All samples were immediately frozen in liquid nitrogen and stored at $-80{ }^{\circ} \mathrm{C}$.

\section{RNA extraction and qRT-PCR analysis}

Total RNA of collected samples was extracted using the Tiangen RNAprep Pure Plant kit (Tiangen, China) according to the manufacturer's instructions. First-strand cDNA was synthesized via reverse transcription of $1 \mu \mathrm{g}$ of total RNA using the PrimeScript RT Reagent kit (Takara, Japan). Primer 5.0 software was used to design the gene-specific primers for qRT-PCR (Additional file 9: Table S7). The histone-3 gene (AF024716) was employed as an internal reference control. The qRT-PCR experiments were performed using SYBR Premix Ex Taq (Takara) on an ABI 7500 real-time PCR system (Applied Biosystems, USA) with three replicates. The details of the protocol were as follows: (Step 1) initial denaturation step of $30 \mathrm{~s}$ at $95^{\circ} \mathrm{C}$, (Step 2) 40 cycles of $5 \mathrm{~s}$ at $95^{\circ} \mathrm{C}$, $34 \mathrm{~s}$ at $60{ }^{\circ} \mathrm{C}$ and (Step 3) melting curve analysis. The $2^{-\triangle \triangle C T}$ method was used to calculated the relative expression levels of GhPELs [75]. T-tests were employed for statistical analyses.

\section{Additional files}

Additional file 1: Table S1. Detailed parameters of the PEL proteins from three cotton species. (XLS $21 \mathrm{~kb}$ )

Additional file 2: Table S2. Numbers of PELs in the five subfamilies in different species. (XLS $9 \mathrm{~kb}$ )

Additional file 3: Table S3. Chromosome locations of PEL genes in three cotton species. (XLS $50 \mathrm{~kb}$ )

Additional file 4: Table S4. Gene duplication events of PEL genes in three cotton species. (XLS $11 \mathrm{~kb}$ )

Additional file 5: Table S5. Ka/Ks analysis and divergence times of segmentally duplicated PEL gene pairs of three cotton species. (XLS $31 \mathrm{~kb}$ )

Additional file 6: Figure S1. The conserved Pec_lyase_C domain and signal peptide of GhPEL proteins. Left: Phylogenetic analysis of GhPEL proteins using MEGA 6.0 via the neighbor-joining (NJ) method with 1,000 bootstrap replicates. Right: Conserved domains of GhPEL proteins. Light-blue filled boxes represent the Pec_lyase_C domain, and green filled boxes represent the signal peptide. (TIF $1224 \mathrm{~kb}$ ) 
Additional file 7: Figure S2. Conserved amino acid sites of the GhPEL proteins. Multiple alignment analysis of GhPEL proteins. The pink, blue, green and red boxes and asterisks represent the substrate binding sites, $\mathrm{Ca}^{2+}$-binding sites, catalysis site and disulfide bond site, respectively. (TIF $6096 \mathrm{~kb}$ )

Additional file 8: Table S6. Cis-elements related to auxin in the promoters of GhPEL genes. (XLS $50 \mathrm{~kb}$ )

Additional file 9: Table S7. Primer pairs used in qRT-PCR analysis. (XLS $25 \mathrm{~kb})$

\section{Abbreviations}

AA: Amino acid; DPA: Days postanthesis.; FPKM: Fragments per kilobase of transcript per million fragments; Ga: Gossypium arboreum; Gh: Gossypium hirsutum; Gr: Gossypium raimondii; Ka: Nonsynonymous substitution rate; Ks: Synonymous substitution rate; MYA: Million years ago; PEL/PLL: Pectate lyase; PMC: Pollen mother cells; qRT-PCR: Quantitative real-time polymerase chain reaction; WGD: Whole-genome duplication

\section{Acknowledgements}

This study was performed at the State Key Laboratory of Cotton Biology at the Institute of Cotton Research of the Chinese Academy of Agricultural Sciences.

\section{Funding}

This research was support by the National Key Research and Development Program of China (grant No. 2016YFD0101400). The funders had no role in the design of the study, the collection, analysis, and interpretation of data, and in writing the manuscript.

\section{Availability of data and materials}

The genomic databases of three cotton species G. raimondii (JGI_V2.1), G.arboreum (BGI_v2.0) and G. hirsutum acc. TM-1 (NAUNBI_v1.1) are available from the CottonGen website (https://www.cottongen.org/icgi/home). The Arabidopsis genome was obtained from the TAIR 10 (http://www.arabidopsis.org). The transcriptome datasets of $\mathrm{G}$. hirsutum TM-1 is accession under the vailable code SRA: PRJNA248163.

\section{Authors' contribution}

SXY, HLW and HTW designed the experiments. YQ, JJS and MZ cultivated the cotton plants in the field and obtained the fiber and anther samples. HRS, QM, LJG and NHW obtained the leaf samples with IAA treatment in the climate-controlled greenhouse. HRS performed the qRT-PCR experiments. HRS, PBH and GYL performed the data analysis. HRS wrote the manuscript. HLW and HTW revised the manuscript. All authors reviewed and approved the final manuscript.

\section{Ethics approval and consent to participate} Not applicable.

\section{Consent for publication}

Not applicable.

\section{Competing interests}

The authors declare that they have no competing interests.

\section{Publisher's Note}

Springer Nature remains neutral with regard to jurisdictional claims in published maps and institutional affiliations.

\section{Received: 11 April 2018 Accepted: 30 August 2018}

Published online: 10 September 2018

\section{References}

1. Leng Y, Yang Y, Ren D, Huang L, Dai L, Wang Y, Chen L, Tu Z, Gao Y, Li X, et al. A Rice PECTATE LYASE-LIKE gene is required for plant growth and leaf senescence. Plant Physiol. 2017;174(2):1151-66.

2. Chebli Y, Geitmann A. Cellular growth in plants requires regulation of cell wall biochemistry. Curr Opin Cell Biol. 2017;44:28-35.
3. Ridley BL, O'Neill MA, Mohnen D. Pectins: structure, biosynthesis, and oligogalacturonide-related signaling. Phytochemistry. 2001;57:929-67.

4. Caffall KH, Mohnen D. The structure, function, and biosynthesis of plant cell wall pectic polysaccharides. Carbohydr Res. 2009;344(14):1879-900.

5. Yadav S, Yadav PK, Yadav D, Yadav KDS. Pectin lyase: a review. Process Biochem. 2009:44(1):1-10.

6. Jimenez-Bermudez S, Redondo-Nevado J, Munoz-Blanco J, Caballero JL, Lopez-Aranda JM, Valpuesta V, Pliego-Alfaro F, Quesada MA, Mercado JA. Manipulation of strawberry fruit softening by antisense expression of a pectate lyase gene. Plant Physiol. 2002;128(2):751-9.

7. Solbak Al, Richardson TH, McCann RT, Kline KA, Bartnek F, Tomlinson G, Tan X, Parra-Gessert L, Frey GJ, Podar M, et al. Discovery of pectin-degrading enzymes and directed evolution of a novel pectate lyase for processing cotton fabric. J Biol Chem. 2005;280(10):9431-8.

8. Zucker M, Hankin L. Regulation of pectate lyase synthesis in Pseudomonas fluorescens and Erwinia carotovora. J Bacteriol. 1970;104(1):13-8.

9. Wing RA, Yamaguchi J, Larabell SK, Ursin VM, McCormick S. Molecular and genetic characterization of two pollen-expressed genes that have sequence similarity to pectate lyases of the plant pathogen Enwinia. Plant Mol Biol. 1989;14:17-28.

10. Kulikauskas R, McCormick S. Identification of the tobacco and Arabidopsis homologues of the pollen-expressed LAT59 gene of tomato. Plant Mol Biol. 1997;34(5):809-14.

11. Taniguchi Y, Ono A, Sawalani M, Nanba M, Kohno K, Usui M, Kunmolo M, Maluhasj T. Cry j l, a major allergen of Japanese cedar pollen, has pectate lyase enzyme activity. Allergy. 1995;50:90-3.

12. Medina-Escobar N, Cardenas J, Moyano E, Caballero JL, Munoz-Blanco J. Cloning, molecular characterization and expression pattern of a strawberry ripening-specific cDNA with sequence homology to pectate lyase from higher plants. Plant Mol Biol. 1997;34:867-77.

13. Payasi A, Sanwal GG. Pectate lyase activity during ripening of banana fruit. Phytochemistry. 2003;63(3):243-8.

14. Yang $L$, Huang $W$, Xiong F, Xian Z, Su D, Ren M, Li Z. Silencing of SIPL, which encodes a pectate lyase in tomato, confers enhanced fruit firmness, prolonged shelf-life and reduced susceptibility to grey mould. Plant Biotechnol J. 2017;15(12):1544-55.

15. Milioni D, Sado PE, Stacey NJ, Domingo C, Roberts K, MC MC. Differential expression of cell-wall-related genes during the formation of tracheary elements in the Zinnia mesophyll cell system. Plant Mol Biol. 2001;47:221-38.

16. Wang H, Guo Y, Lv F, Zhu H, Wu S, Jiang Y, Li F, Zhou B, Guo W, Zhang T. The essential role of GhPEL gene, encoding a pectate lyase, in cell wall loosening by depolymerization of the de-esterified pectin during fiber elongation in cotton. Plant Mol Biol. 2010;72(4-5):397-406.

17. Bai Y, Wu D, Liu F, Li Y, Chen P, Lu M, Zheng B. Characterization and functional analysis of the poplar Pectate Lyase-like gene PtPL1-18 reveal its role in the development of vascular tissues. Front Plant Sci. 2017:8:1123.

18. Laskowski M, Biller S, Stanley K, Kajstura T, Prusty R. Expression profiling of auxin-treated Arabidopsis roots: toward a molecular analysis of lateral root emergence. Plant Cell Physiol. 2006;47(6):788-92.

19. Palusa SG, Golovkin M, Shin SB, Richardson DN, Reddy AS. Organ-specific, developmental, hormonal and stress regulation of expression of putative pectate lyase genes in Arabidopsis. New Phytol. 2007;174(3):537-50.

20. Sun L, van Nocker S. Analysis of promoter activity of members of the PECTATE LYASE-LIKE (PLL) gene family in cell separation in Arabidopsis. BMC Plant Biol. 2010;10:152.

21. Cosgrove DJ. Growth of the plant cell wall. Nat Rev Mol Cell Biol. 2005;6(11):850-61

22. Heisler MG, Ohno C, Das P, Sieber P, Reddy GV, Long JA, Meyerowitz EM. Patterns of auxin transport and gene expression during primordium development revealed by live imaging of the Arabidopsis inflorescence meristem. Curr Biol. 2005;15(21):1899-911.

23. Payasi A, Misra PC, Sanwal GG. Effect of phytohormones on pectate lyase activity in ripening Musa acuminata. Plant Physiol Biochem. 2004;42(11):861-5.

24. Domingo C, Roberts K, Stacey NJ, Connerton I, Ruiz-Teran F, McCann MC. A pectate lyase from Zinnia elegans is auxin inducible. Plant J. 1998;13:17-28.

25. Anderson DB: Growth and structure of cotton fiber. January 1938, 30(1): 48-54

26. Meinert MC, Delmer DP. Changes in biochemical composition of the cell wall of the cotton fiber during development. Plant Physiology. 1977;59:1088-97.

27. Paterson AH, Wendel JF, Gundlach H, Guo H, Jenkins J, Jin D, Llewellyn D, Showmaker KC, Shu S, Udall J, et al. Repeated polyploidization of Gossypium genomes and the evolution of spinnable cotton fibres. Nature. 2012;492(7429):423-7. 
28. Wang K, Wang Z, Li F, Ye W, Wang J, Song G, Yue Z, Cong L, Shang H, Zhu $S$, et al. The draft genome of a diploid cotton Gossypium raimondii. Nat Genet. 2012;44(10):1098-103.

29. Li F, Fan G, Wang K, Sun F, Yuan Y, Song G, Li Q, Ma Z, Lu C, Zou C, et al. Genome sequence of the cultivated cotton Gossypium arboreum. Nat Genet. 2014;46(6):567-72

30. Zhang T, Hu Y, Jiang W, Fang L, Guan X, Chen J, Zhang J, Saski CA, Scheffler BE, Stelly DM, et al. Sequencing of allotetraploid cotton (Gossypium hirsutum L. acc. TM-1) provides a resource for fiber improvement. Nat Biotechnol. 2015;33(5):531-7.

31. Li F, Fan G, Lu C, Xiao G, Zou C, Kohel RJ, Ma Z, Shang H, Ma X, Wu J, et al. Genome sequence of cultivated upland cotton (Gossypium hirsutum TM-1) provides insights into genome evolution. Nat Biotechnol. 2015;33(5):524-30.

32. Cannon SB, Mitra A, Baumgarten A, Young ND, May G. The roles of segmental and tandem gene duplication in the evolution of large gene families in Arabidopsis thaliana. BMC Plant Biol. 2004;4:10

33. Wolf $\mathrm{S}$, Hematy $\mathrm{K}$, Hofte $\mathrm{H}$. Growth control and cell wall signaling in plants. Annu Rev Plant Biol. 2012;63:381-407.

34. Su J, Li L, Pang C, Wei H, Wang C, Song M, Wang H, Zhao S, Zhang C, Mao $G$, et al. Two genomic regions associated with fiber quality traits in Chinese upland cotton under apparent breeding selection. Sci Rep. 2016;6:38496.

35. Davis KR, Lyon GD, Darvill AG, Albersheim P. Host-pathogen interactions. Plant Physiol. 1984:74:52-60.

36. Jiang J, Yao L, Miao Y, Cao J. Genome-wide characterization of the Pectate Lyase-like (PLL) genes in Brassica rapa. Mol Genet Genomics. 2013;288(11):601-14

37. Moore RC, Purugganan MD. The evolutionary dynamics of plant duplicate genes. Curr Opin Plant Biol. 2005;8(2):122-8.

38. Flagel LE, Wendel JF. Gene duplication and evolutionary novelty in plants. The New phytologist. 2009;183(3):557-64.

39. Li W, Shang H, Ge Q, Zou C, Cai J, Wang D, Fan S, Zhang Z, Deng X, Tan Y, et al. Genome-wide identification, phylogeny, and expression analysis of pectin methylesterases reveal their major role in cotton fiber development. BMC Genomics. 2016:17(1):1000.

40. Wang X, Ma Q, Dou L, Liu Z, Peng R, Yu S. Genome-wide characterization and comparative analysis of the MLO gene family in cotton. Plant Physiol Biochem. 2016;103:106-19.

41. He X, Zhang J. Rapid subfunctionalization accompanied by prolonged and substantial neofunctionalization in duplicate gene evolution. Genetics. 2005:169(2):1157-64.

42. Willats WGT, McCartney L, Mackie W, Knox JP. Pectin: cell biology and prospects for functional analysis. Plant Mol Biol. 2001;47(1/2):9-27.

43. Francis KE, Lam SY, Copenhaver GP. Separation of Arabidopsis pollen tetrads is regulated by QUARTET1, a pectin methylesterase gene. Plant Physiol. 2006;142(3):1004-13.

44. Rhee SY, Somerville CR. Tetrad pollen formation in quartet mutants of Arabidopsis thaliana is associated with persistence of pectic polysaccharides of the pollen mother cell wall. Plant J. 1998;15(1):79-88.

45. Blackmore S, Wortley AH, Skvarla JJ, Rowley JR. Pollen wall development in flowering plants. New Phytol. 2007;174(3):483-98.

46. Jiang J, Zhang Z, Cao J. Pollen wall development: the associated enzymes and metabolic pathways. Plant Biol. 2013;15(2):249-63.

47. Wilkins TA, Rajasekaran K, Anderson DM. Cotton biotechnology. Crit Rev Plant Sci. 2010;19(6):511-50.

48. Hu H, He X, Tu L, Zhu L, Zhu S, Ge Z, Zhang X. GhJAZ2 negatively regulates cotton fiber initiation by interacting with the R2R3-MYB transcription factor GhMYB25-like. Plant J. 2016;88(6):921-35.

49. Suo J, Liang X, Pu L, Zhang Y, Xue Y. Identification of GhMYB109 encoding a R2R3 MYB transcription factor that expressed specifically in fiber initials and elongating fibers of cotton (Gossypium hirsutum L.). Biochi Biophys Acta. 2003:1630(1):25-34.

50. Bohn-Courseau I. Auxin: a major regulator of organogenesis. C R Biol. 2010:333(4):290-6.

51. Singh B, Cheek HD, Haigler CH. A synthetic auxin (NAA) suppresses secondary wall cellulose synthesis and enhances elongation in cultured cotton fiber. Plant Cell Rep. 2009;28(7):1023-32.

52. Cecchetti V, Brunetti P, Napoli N, Fattorini L, Altamura MM, Costantino P, Cardarelli M. ABCB1 and ABCB19 auxin transporters have synergistic effects on early and lateArabidopsisanther development. J Integr Plant Biol. 2015; 57(12):1089-98.
53. Cecchetti V, Altamura MM, Falasca G, Costantino P, Cardarelli M. Auxin regulates Arabidopsis anther dehiscence, pollen maturation, and filament elongation. Plant Cell. 2008;20(7):1760-74.

54. Wolf S, Mouille G, Pelloux J. Homogalacturonan methyl-esterification and plant development. Mol Plant. 2009;2(5):851-60.

55. Finn RD, Coggill P, Eberhardt RY, Eddy SR, Mistry J, Mitchell AL, Potter SC, Punta M, Qureshi M, Sangrador-Vegas A, et al. The Pfam protein families database: towards a more sustainable future. Nucleic Acids Res. 2016:44(D1):D279-85.

56. Yu J, Jung S, Cheng CH, Ficklin SP, Lee T, Zheng P, Jones D, Percy RG, Main D. CottonGen: a genomics, genetics and breeding database for cotton research. Nucleic Acids Res. 2014;42(Database issue):D1229-36.

57. Letunic I, Doerks T, Bork P. SMART: recent updates, new developments and status in 2015. Nucleic Acids Res. 2015;43(Database issue):D257-60.

58. Artimo P, Jonnalagedda M, Arnold K, Baratin D, Csardi G, de Castro E, Duvaud S, Flegel V, Fortier A, Gasteiger E, et al. ExPASy: SIB bioinformatics resource portal. Nucleic acids research. 2012;40(Web Server issue):W597-603.

59. Yu CS, Lin CJ, Hwang JK. Predicting subcellular localization of proteins for gram-negative bacteria by support vector machines based on n-peptide compositions. Protein Sci. 2004;13(5):1402-6.

60. Li X, Liu G, Geng Y, Wu M, Pei W, Zhai H, Zang X, Li X, Zhang J, Yu S, et al. A genome-wide analysis of the small auxin-up RNA (SAUR) gene family in cotton. BMC Genomics. 2017;18(1):815.

61. Tamura K, Stecher G, Peterson D, Filipski A, Kumar S. MEGA6: molecular evolutionary genetics analysis version 6.0. Mol Biol Evol. 2013;30(12):2725-9.

62. Wei F, Coe E, Nelson W, Bharti AK, Engler F, Butler E, Kim H, Goicoechea JL, Chen M, Lee S et al: Physical and Genetic Structure of the Maize Genome Reflects its Complex Evolutionary History. PLoS Genetics 2005, preprint(2007):e123.

63. Zhang $X$, Feng $Y$, Cheng $H$, Tian D, Yang S, Chen JQ. Relative evolutionary rates of NBS-encoding genes revealed by soybean segmental duplication. Mol Genet Genomics. 2011;285(1):79-90.

64. Wang LQ, Guo K, Li Y, Tu YY, Hu HZ, Wang BR, Cui XC. Expression profiling and integrative analysis of CESA/CSL superfamily in rice. BMC Plant Biol. 2010;10:282-97.

65. Krzywinski M, Schein J, Birol I, Connors J, Gascoyne R, Horsman D, Jones SJ, Marra MA. Circos: an information aesthetic for comparative genomics. Genome Res. 2009;19(9):1639-45.

66. Librado P, Rozas J. DnaSP v5: a software for comprehensive analysis of DNA polymorphism data. Bioinformatics. 2009;25(11):1451-2.

67. Yang X, Tuskan GA, Cheng MZ. Divergence of the Dof gene families in poplar, Arabidopsis, and rice suggests multiple modes of gene evolution after duplication. Plant Physiol. 2006;142(3):820-30.

68. Petersen TN, Brunak S, von Heijne G, Nielsen H. SignalP 4.0: discriminating signal peptides from transmembrane regions. Nat Methods. 2011;8(10):785-6

69. Hu B, Jin J, Guo A-Y, Zhang H, Luo J, Gao G. GSDS 2.0: an upgraded gene feature visualization server. Bioinformatics. 2015;31(8):1296-7.

70. Bailey TL, Williams N, Misleh C, Li WW. MEME: discovering and analyzing DNA and protein sequence motifs. Nucleic Acids Res. 2006;34(Web Server issue):W369-73.

71. Vogel C, Marcotte EM. Insights into the regulation of protein abundance from proteomic and transcriptomic analyses. Nat Rev Genet. 2012;13(4):227-32.

72. Hebenstreit D, Fang M, Gu M, Charoensawan V, van Oudenaarden A, Teichmann SA. RNA sequencing reveals two major classes of gene expression levels in metazoan cells. Mol Syst Biol. 2011;7:497.

73. Gonzalez-Porta M, Frankish A, Rung J, Harrow J, Brazma A. Transcriptome analysis of human tissues and cell lines reveals one dominant transcript per gene. Genome Biol. 2013;14(7):R70.

74. Liu J, Pang C, Wei H, Song M, Meng Y, Ma J, Fan S, Yu S. iTRAQ-facilitated proteomic profiling of anthers from a photosensitive male sterile mutant and wild-type cotton (Gossypium hirsutum L.). J Proteome. 2015:126:68-81.

75. Li X, Wu M, Liu G, Pei W, Zhai H, Yu J, Zhang J, Yu S. Identification of candidate genes for fiber length quantitative trait loci through RNA-Seq and linkage and physical mapping in cotton. BMC Genomics. 2017;18(1):427. 